PROCEEDINGS OF THE

AMERICAN MATHEMATICAL SOCIETY

Volume 138, Number 3, March 2010, Pages 937-942

S 0002-9939(09)10182-X

Article electronically published on October 20, 2009

\title{
PROOF OF A DYNAMICAL BOGOMOLOV CONJECTURE FOR LINES UNDER POLYNOMIAL ACTIONS
}

\author{
DRAGOS GHIOCA AND THOMAS J. TUCKER \\ (Communicated by Ted Chinburg)
}

\begin{abstract}
We prove a dynamical version of the Bogomolov conjecture in the special case of lines in $\mathbb{A}^{m}$ under the action of a map $\left(f_{1}, \ldots, f_{m}\right)$, where each $f_{i}$ is a polynomial in $\overline{\mathbb{Q}}[X]$ of the same degree.
\end{abstract}

\section{INTRODUCTION}

In 1998, Ullmo [Ul198] and Zhang [Zha98] proved the following theorem, which had been conjectured by Bogomolov Bog91.

Theorem 1.1. Let $A$ be an abelian variety defined over a number field with NéronTate height $\widehat{h}_{\mathrm{nt}}$ and let $W$ be an irreducible subvariety of $A$ that is not a torsion translate of an abelian subvariety of $A$. Then there exists an $\epsilon>0$ such that the set

$$
\left\{x \in A(\overline{\mathbb{Q}}) \mid \widehat{h}_{\mathrm{nt}}(x) \leq \epsilon\right\}
$$

is not Zariski dense in $W$.

Earlier, Zhang [Zha95a] had proved a similar result for the multiplicative group $\mathbb{G}_{m}^{n}$. Zhang Zha95b, Zha06 also proposed a more general conjecture for what he called polarizable morphisms: a morphism $\Phi: X \longrightarrow X$ on a projective variety $X$ is said to be polarizable if there is an ample line bundle $\mathcal{L}$ on $X$ such that $\Phi^{*} \mathcal{L} \cong q \mathcal{L}$ for some integer $q>1$. When a polarizable map $\Phi$ is defined over a number field, it gives rise to a canonical height $\widehat{h}_{\Phi}$ with the property that $\widehat{h}_{\Phi}(\Phi(\alpha))=q \widehat{h}_{\Phi}(\alpha)$ for all $\alpha \in X(\overline{\mathbb{Q}})$. Zhang makes the following Bogomolov-type conjecture in this more general context.

Conjecture 1.2 (Zhang). Let $\Phi: X \longrightarrow X$ be a polarizable morphism of a projective variety defined over a number field and let $W$ be a subvariety of $X$ that is not preperiodic under $\Phi$. Then there exists an $\epsilon>0$ such that the set

$$
\left\{x \in W(\overline{\mathbb{Q}}) \mid \widehat{h}_{\Phi}(x) \leq \epsilon\right\}
$$

is not Zariski dense in $W$.

Received by the editors October 22, 2008, and, in revised form, May 1, 2009.

2010 Mathematics Subject Classification. Primary 37P05; Secondary 14G25, 11C08.

The first author was partially supported by NSERC.

The second author was partially supported by NSA Grant 06G-067 and NSF Grant DMS0801072.

(C)2009 American Mathematical Society 
The definition of preperiodicity for varieties here is the same as the usual definition of preperiodicity for points. More precisely, for any quasiprojective variety $X$, any endomorphism $\Phi: X \longrightarrow X$, and any subvariety $V \subset X$, we say that $V$ is $\Phi$-preperiodic if there exists $N \geq 0$, and $k \geq 1$ such that $\Phi^{N}(V)=\Phi^{N+k}(V)$. Note that when $A$ is an abelian variety and $\Phi$ is a multiplication-by- $n$ map (for $n \geq 2$ ), an irreducible subvariety $W$ is preperiodic if and only if it is a torsion translate of an abelian subvariety of $A$.

We note that if $X=\left(\mathbb{P}^{1}\right)^{n}$ and if $\Phi$ is given by the coordinatewise action of $z^{d}$ (for some $d \geq 2$ ) on $X$, then Conjecture 1.2 reduces to the result proved by Zhang in Zha95a.

In this paper, we prove the following special case of Conjecture 1.2

Theorem 1.3. Let $f_{1}, \ldots, f_{m} \in \overline{\mathbb{Q}}[X]$ be polynomials of degree $d>1$, let $\Phi:=$ $\left(f_{1}, \ldots, f_{m}\right)$ be their coordinatewise action on $\mathbb{A}^{m}$, and let $L$ be a line in $\mathbb{A}^{m}$ defined over $\overline{\mathbb{Q}}$. If $L$ is not $\Phi$-preperiodic, then there exists an $\epsilon>0$ such that

$$
S_{L, \Phi, \epsilon}:=\left\{x \in L(\overline{\mathbb{Q}}) \mid \widehat{h}_{\Phi}(x) \leq \epsilon\right\}
$$

is finite (see Section 2 for the definition of $\widehat{h}_{\Phi}$ ).

Baker and Hsia [BH05, Theorem 8.10] previously proved Theorem 1.3 in the special case where $f_{1}=f_{2}$ and $m=2$.

In Section 2 we introduce our notation for canonical heights associated to polynomials. Then in Section 3 we present some general results regarding polynomials which share the same Julia set. In Section 4 we prove Theorem 1.3 and then describe a few examples of preperiodic lines under the coordinatewise action of polynomials of the same degree.

\section{Preliminaries}

Heights. Let $\mathbb{M}_{\mathbb{Q}}$ be the usual set of absolute values on $\mathbb{Q}$, normalized so that the Archimedean absolute value is simply the absolute value $|\cdot|$ and $|p|_{p}=1 / p$ for each $p$-adic absolute value $|\cdot|_{p}$. For any finite extension $K$ of $\mathbb{Q}$ we define $\mathbb{M}_{K}$ to be the set of absolute values on $K$ that extend elements of $\mathbb{M}_{\mathbb{Q}}$. Then, for any $x \in \overline{\mathbb{Q}}$ we define the Weil height of $x$ to be

$$
h(x)=\frac{1}{[\mathbb{Q}(x): \mathbb{Q}]} \cdot \sum_{v \in \mathbb{M}_{\mathbb{Q}}} \sum_{\substack{w \mid v \\ w \in \mathbb{M}_{\mathbb{Q}(x)}}} \log \max \left\{|x|_{w}^{\left[\mathbb{Q}(x)_{w}: \mathbb{Q}_{v}\right]}, 1\right\},
$$

where $\mathbb{Q}_{v}$ and $\mathbb{Q}(x)_{w}$ are the completions of $\mathbb{Q}$ and $\mathbb{Q}(x)$ at $v$ and $w$ respectively (see [BG06, Chapter 1] for details).

For a polynomial $f \in \overline{\mathbb{Q}}[X]$ of degree greater than 1 , define the $f$-canonical height $\widehat{h}_{f}: \overline{\mathbb{Q}} \longrightarrow \mathbb{R}_{\geq 0}$ by

$$
\widehat{h}_{f}(x)=\lim _{n \rightarrow \infty} \frac{h\left(f^{n}(x)\right)}{(\operatorname{deg} f)^{n}},
$$

following Call-Silverman CS93] (where $f^{n}$ denotes the $n$-th iterate of $f$ ).

Let $f_{1}, \ldots, f_{m} \in \overline{\mathbb{Q}}[X]$ be polynomials of degree $d>1$, and let $\Phi:=\left(f_{1}, \ldots, f_{m}\right)$ be their coordinatewise action on $\mathbb{A}^{m}$; that is,

$$
\Phi\left(x_{1}, \ldots, x_{m}\right)=\left(f_{1}\left(x_{1}\right), \ldots, f_{m}\left(x_{m}\right)\right) .
$$


We define the $\Phi$-canonical height $\widehat{h}_{\Phi}: \mathbb{A}^{m}(\overline{\mathbb{Q}}) \longrightarrow \mathbb{R}_{\geq 0}$ by

$$
\widehat{h}_{\Phi}\left(x_{1}, \ldots, x_{m}\right)=\sum_{i=1}^{m} \widehat{h}_{f_{i}}\left(x_{i}\right) \text {. }
$$

Note that while $\mathbb{A}^{m}$ is not a projective variety, $\Phi$ extends to a map $\tilde{\Phi}:\left(\mathbb{P}^{1}\right)^{m} \longrightarrow$ $\left(\mathbb{P}^{1}\right)^{m}$. Furthermore, $\tilde{\Phi}$ is polarizable, since

$$
\tilde{\Phi}^{*} \bigotimes_{i=1}^{m} \operatorname{pr}_{i}^{*} \mathcal{O}_{\mathbb{P}^{1}}(1) \cong \bigotimes_{i=1}^{m} \operatorname{pr}_{i}^{*} \mathcal{O}_{\mathbb{P}^{1}}(d),
$$

where $\operatorname{pr}_{i}$ is the projection of $\left(\mathbb{P}^{1}\right)^{m}$ onto its $i$-th coordinate.

Remark. Theorem 1.3 is not true if one allows the polynomials $f_{i}$ to have different degrees. This is easily seen, for example, in the case where $m=2$, the line $L$ is the diagonal, and $f_{2}=f_{1}^{2}$. The map $\Phi=\left(f_{1}, \ldots, f_{m}\right)$ is only polarizable when $\operatorname{deg} f_{i}=\operatorname{deg} f_{j}$, so this is not a counterexample to Conjecture 1.2 .

\section{Symmetries of the Julia Set}

In this section we recall the main results regarding polynomials which share the same Julia set. For a polynomial $f \in \mathbb{C}[X]$, we let $J(f)$ denote the Julia set of $f$ (see [Bea91, Chapter 3] or Mil99 for the definition of a Julia set of a rational function over the complex numbers). As proved by Beardon [Bea90, Bea92, any family of polynomials which have the same Julia set $\mathcal{J}$ is determined by the symmetries of $\mathcal{J}$.

Definition 3.1. If $f \in \mathbb{C}[z]$ is a polynomial and $J(f)$ is its Julia set, then the symmetry group $\Sigma(f)$ of $J(f)$ is defined by

$$
\Sigma(f)=\{\sigma \in \mathfrak{C}: \sigma(J(f))=J(f)\},
$$

where $\mathfrak{C}$ is the group of conformal Euclidean isometries.

Beardon [Bea90, Lemma 3] computed $\Sigma(f)$ for any $f \in \mathbb{C}[z]$.

Lemma 3.2. The isometry group $\Sigma(f)$ is a group of rotations about some point $\zeta \in \mathbb{C}$, and it is either trivial or a finite cyclic group or the group of all rotations about $\zeta$.

To prove the result above, Beardon uses the fact that each polynomial $f$ of degree $d \geq 2$ is conjugate to a monic polynomial $\tilde{f} \in \mathbb{C}[z]$ which has no term in $z^{d-1}$. Clearly, if $\tilde{f}=\gamma \circ f \circ \gamma^{-1}$, where $\gamma \in \mathbb{C}[z]$ is a linear polynomial, then $J(\tilde{f})=\gamma(J(f))$; thus it suffices to prove Lemma 3.2 for $\tilde{f}$ instead of $f$. If $\tilde{f}(z)=z^{d}$, then $J(\tilde{f})$ is the unit circle, and $\Sigma(\tilde{f})$ is the group of all rotations about 0 (see also Bea90, Lemma 4]). If $\tilde{f}$ is not a monomial, then we choose $b \geq 1$ maximal such that we can find $a \geq 0$ and $\tilde{f}_{1} \in \mathbb{C}[z]$ satisfying $\tilde{f}(z)=z^{a} \tilde{f}_{1}\left(z^{b}\right)$. In this case, $J(\tilde{f})$ is the finite cyclic group of rotations generated by the multiplication by $\exp (2 \pi i / b)$ on $\mathbb{C}$ (see Bea90, Theorem 5]). Beardon also proves the following result (see Bea90, Lemma 7]), which we will use later.

Lemma 3.3. If $f \in \mathbb{C}[z]$ is a polynomial of degree $d \geq 2$ and $\sigma \in J(f)$, then $f \circ \sigma=\sigma^{d} \circ f$.

The following classification of polynomials which have the same Julia set is proved by Beardon in Bea92, Theorem 1]. 
Lemma 3.4. If $f, g \in \mathbb{C}[z]$ have the same Julia set, then there exists $\sigma \in \Sigma(f)$ such that $g=\sigma \circ f$.

\section{Proof of our main Result}

Proof of Theorem 1.3. Suppose that for every $\epsilon>0$, the set $S_{L, \Phi, \epsilon}$ is infinite. We will show that this implies that $L$ must be $\Phi$-preperiodic.

We first note that it suffices to prove the theorem for the line $L^{\prime}=\left(\sigma_{1}, \ldots, \sigma_{m}\right)(L)$ and the map

$$
\Phi^{\prime}=\left(\sigma_{1} \circ f_{1} \circ \sigma_{1}^{-1}, \ldots, \sigma_{m} \circ f_{m} \circ \sigma_{m}^{-1}\right)
$$

for some linear automorphisms $\sigma_{1}, \ldots, \sigma_{m}$ of $\mathbb{A}^{1}$. This follows from the fact that $L$ is preperiodic for $\Phi$ if and only if $L^{\prime}$ is preperiodic for $\Phi^{\prime}$ along with the equality

$$
\widehat{h}_{\Phi^{\prime}}\left(\sigma_{1} \alpha_{1}, \ldots, \sigma_{m} \alpha_{m}\right)=\widehat{h}_{\Phi}\left(\alpha_{1}, \ldots, \alpha_{m}\right) .
$$

Note that (4.1) is a simple consequence of Definition 2.1. since $\left|h\left(\sigma_{i} x\right)-h(x)\right|$ is bounded for all $x \in \overline{\mathbb{Q}}$.

We now proceed by induction on $m$; the case $m=1$ is obvious.

If the projection of $L$ on any of the coordinates consists of only one point, we are done by the inductive hypothesis. Indeed, without loss of generality, assume the projection of $L$ on the first coordinate equals $\left\{z_{1}\right\}$. Then $L=\left\{z_{1}\right\} \times L_{1}$, where $L_{1} \subset \mathbb{A}^{m-1}$ is a line, and $\widehat{h}_{f_{1}}\left(z_{1}\right)=0$. Since only preperiodic points have canonical height equal to 0 (see [CS93, Cor. 1.1.1]), we conclude that $z_{1}$ is $f_{1}$-preperiodic, and thus we are done by the induction hypothesis applied to $L_{1}$.

Suppose now that $L$ projects dominantly onto each coordinate of $\mathbb{A}^{m}$. For each $i=2, \ldots, m$, we let $L_{i}$ be the projection of $L$ on the first and the $i$-th coordinates of $\mathbb{A}^{m}$. Then $L_{i}$ is a line given by an equation $X_{1}=\sigma_{i}\left(X_{i}\right)$, for some linear polynomial $\sigma_{i} \in \overline{\mathbb{Q}}[X]$. Clearly, it suffices to show that for each $i=2, \ldots, m$, the line $L_{i}$ is preperiodic under the action of $\left(f_{1}, f_{i}\right)$ on the corresponding two coordinates of $\mathbb{A}^{m}$. Indeed, if for each $i=2, \ldots, m$, we show that there exist $a_{i}, b_{i} \in \mathbb{N}$ (with $a_{i}<b_{i}$ ) such that $\left(f_{1}^{a_{i}}, f_{i}^{a_{i}}\right)\left(L_{i}\right)=\left(f_{1}^{b_{i}}, f_{i}^{b_{i}}\right)\left(L_{i}\right)$, then

$$
\left(f_{1}^{a}, \ldots, f_{m}^{a}\right)(L)=\left(f_{1}^{a+b}, \ldots, f_{m}^{a+b}\right)(L)
$$

where $a:=\max _{i=2}^{m} a_{i}$, and $b$ is the least common multiple of all $\left(b_{i}-a_{i}\right)$ for $i=2, \ldots, m$.

Let $\tilde{f}_{i}:=\sigma_{i} \circ f_{i} \circ \sigma_{i}^{-1}$ and let $\Delta=(x, x) \in \mathbb{A}^{2}$ be the diagonal on $\mathbb{A}^{2}$. By our remarks at the beginning of the proof, it suffices to show that (id, $\left.\sigma_{i}\right)\left(L_{i}\right)=\Delta$ is preperiodic under the action of $\left(f_{1}, \tilde{f}_{i}\right)$. Furthermore, the fact that we have an infinite sequence $\left(z_{n, 1}, z_{n, i}\right) \in L_{i}(\overline{\mathbb{Q}})$ with

$$
\lim _{n \rightarrow \infty} \widehat{h}_{f_{1}}\left(z_{n, 1}\right)=\lim _{n \rightarrow \infty} \widehat{h}_{f_{i}}\left(z_{n, i}\right)=0
$$

implies that we have

$$
\lim _{n \rightarrow \infty} \widehat{h}_{\tilde{f}_{i}}\left(z_{n, 1}\right)=0
$$

because of (4.1). Fix an embedding $\theta: \overline{\mathbb{Q}} \longrightarrow \mathbb{C}$ and let $f_{1}^{\theta}$ and $\tilde{f}_{i}^{\theta}$ be the images of $f_{1}$ and $\tilde{f}_{i}$, respectively, in $\mathbb{C}[X]$ under this embedding. Then, by BH05, Corollary 4.6], the Galois orbits of the points $\left\{z_{n, 1}\right\}_{n \in \mathbb{N}}$ are equidistributed with respect to the equilibrium measures on the Julia sets of both $f_{1}^{\theta}$ and $\tilde{f}_{i}^{\theta}$. Since the support of the equilibrium measure $\mu_{g}$ of a polynomial $g \in \mathbb{C}[X]$ is equal to the Julia set of $g\left(\right.$ BH05, Section 4]), we must have $J\left(\tilde{f}_{i}^{\theta}\right)=J\left(f_{1}^{\theta}\right)$. 
By Lemma 3.4 (see also BE87, AH96]), there exists a conformal Euclidean symmetry $\mu_{i}: z \longrightarrow a_{i} z+b_{i}$ such that $\mu_{i}\left(J\left(f_{1}^{\theta}\right)\right)=J\left(f_{1}^{\theta}\right)$ and $\tilde{f}_{i}^{\theta}=\mu_{i} \circ f_{1}^{\theta}$. Note that $a_{i}$ and $b_{i}$ must be in the image of $\overline{\mathbb{Q}}$ under $\theta$ since the coefficients of $f_{1}^{\theta}$ and $f_{i}^{\theta}$ are. Let $\tau_{i}$ be the map $\tau_{i}: z \longrightarrow \theta^{-1}\left(a_{i}\right) z+\theta^{-1}\left(b_{i}\right)$. Then we have $\tilde{f}_{i}=\tau_{i} \circ f_{1}$.

If $\tau_{i}$ has infinite order, then it follows from [Bea90, Lemma 4] that there exist linear polynomials $\gamma_{1}, \gamma_{i}$ such that $\gamma_{1} \circ f_{1} \circ \gamma_{1}^{-1}=\gamma_{i} \circ \tilde{f}_{i} \circ \gamma_{i}^{-1}=X^{d}$. In this case, we reduce our problem to the usual Bogomolov conjecture for $\mathbb{G}_{m}^{2}$, proved by Zhang Zha92. Indeed, Zhang proves that if a curve $C$ in $\mathbb{G}_{m}^{2}$ has an infinite family of algebraic points with height tending to zero, then it must be a torsion translate of an algebraic subgroup of $\mathbb{G}_{m}^{2}$; that is, $C=\xi A$ where $\xi$ has finite order and $A$ is an algebraic subgroup of $\mathbb{G}_{m}^{2}$. Since $(\xi A)^{n}=\xi^{n} A$ and $\xi$ has finite order, it is clear that such a curve is preperiodic under the map $(X, Y) \mapsto\left(X^{d}, Y^{d}\right)$.

We may suppose then that $\tau_{i}$ has finite order. By Lemma 3.3, we have $f_{1} \circ \tau_{i}=$ $\tau_{i}^{d} \circ f_{1}$. Thus, we have

$$
\tilde{f}_{i}^{k}=\tau_{i}^{\left(d^{k}-1\right) /(d-1)} \circ f_{1}^{k}
$$

for all $k \geq 1$. Since $\tau_{i}$ has finite order, we conclude that the set

$$
\left\{\tau_{i}^{\left(d^{k}-1\right) /(d-1)}\right\}_{k \geq 0}
$$

is finite. This implies that the set of curves of the form $\left(f_{1}^{k}, \tilde{f}_{i}{ }^{k}\right)(\Delta)$ is finite, which means that the diagonal subvariety $\Delta$ is preperiodic under the action of $\left(f_{1}, \tilde{f}_{i}\right)$, as desired.

4.1. Examples. Note that $\Delta$ is periodic under $\Phi$ only if there is some $n$ such that $f^{n}=g^{n}$. Take, for example, $f(x)=x^{3}, g(x)=-x^{3}$; then we have $f^{2}=g^{2}$, so $\Delta$ has period two under the action of $\Phi$. More generally, there exists some $n$ such that $f^{n}=g^{n}$ if and only if $f(x)=-\beta+\gamma h(x+\beta)$ and $g(x)=-\beta+h(x+\beta)$ for some $\gamma \in \mathbb{C}^{*}, \beta \in \mathbb{C}$ and $h \in x^{r} \mathbb{C}\left[x^{s}\right]$ (with $r, s \geq 0$ ) such that $\gamma^{s}=1$ and $\gamma^{\left(d^{n}-1\right) /(d-1)}=1$ (see GTZ08, Prop. 6.3]). On the other hand, if $f(x)=h\left(x^{s}\right)$ for some positive integer $s \geq 2$ and $\zeta \in \mathbb{C}$ is an $s$-th root of unity and $g(x)=\zeta \cdot f(x)$, then $\Delta$ is $\Phi$-preperiodic, but it is not $\Phi$-periodic.

We believe that it is possible to extend the methods of the proof of Theorem 1.3 to the case of arbitrary rational maps $\varphi_{1}, \ldots, \varphi_{m}$ of the same degree, though the proof seems to be much more difficult, requiring in particular Mimar's Mim97. results on arithmetic intersections of metrized line bundles and an analysis of Douady-Hubbard-Thurston's DH93] classification of critically finite rational maps with parabolic orbifolds. We intend to treat this problem in a future paper.

\section{ACKNOWLEDGMENTS}

We would like to thank Arman Mimar for helpful conversations. We would also like to thank the referee for many useful suggestions.

\section{REFERENCES}

[AH96] P. Atela and J. Hu, Commuting polynomials and polynomials with same Julia set, Internat. J. Bifur. Chaos Appl. Sci. Engrg. 6 (1996), no. 12A, 2427-2432. MR1445904 (98c:58133)

[BE87] I. N. Baker and A. Erëmenko, A problem on Julia sets, Ann. Acad. Sci. Fenn. Ser. A I Math. 12 (1987), no. 2, 229-236. MR 951972 (89g:30047)

[BH05] M. H. Baker and L.-C. Hsia, Canonical heights, transfinite diameters, and polynomial dynamics, J. Reine Angew. Math. 585 (2005), 61-92. MR2164622 (2006i:11071) 
[Bea90] A. F. Beardon, Symmetries of Julia sets, Bull. London Math. Soc. 22 (1990), no. 6, 576-582. MR 1099008 (92f:30033)

[Bea91] A. F. Beardon, Iteration of rational functions, Springer-Verlag, New York, 1991. MR.1128089 (92j:30026)

[Bea92] A. F. Beardon, Polynomials with identical Julia sets, Complex Variables Theory Appl. 17 (1992), no. 3-4, 195-200. MR1147050 (93k:30033)

[BG06] E. Bombieri and W. Gubler, Heights in Diophantine geometry, New Mathematical Monographs, vol. 4, Cambridge University Press, Cambridge, 2006. MR 2216774 (2007a:11092)

[Bog91] F. A. Bogomolov, Abelian subgroups of Galois groups, Izv. Akad. Nauk SSSR Ser. Mat. 55 (1991), no. 1, 32-67. MR.1130027 (93b:12007)

[CS93] G. S. Call and J. Silverman, Canonical heights on varieties with morphism, Compositio Math. 89 (1993), 163-205. MR1255693 (95d:11077)

[DH93] A. Douady and J. H. Hubbard, A proof of Thurston's topological characterization of rational functions, Acta Math. 171 (1993), no. 2, 263-297. MR.1251582 (94j:58143)

[GTZ08] D. Ghioca, T. J. Tucker, and M. E. Zieve, Intersections of polynomials orbits, and a dynamical Mordell-Lang conjecture, Invent. Math. 171 (2008), no. 2, 463-483. MR2367026 (2008k:37099)

[Mil99] J. Milnor, Dynamics in one complex variable, Vieweg, Braunschweig, 1999. MR.1721240 (2002i:37057)

[Mim97] A. Mimar, On the preperiodic points of an endomorphism of $\mathbb{P}^{1} \times \mathbb{P}^{1}$ which lie on a curve, Ph.D. thesis, Columbia University, 1997.

[Ul198] E. Ullmo, Positivité et discrétion des points algébriques des courbes, Ann. of Math. (2) 147 (1998), no. 1, 167-179. MR1609514 (99e:14031)

[Zha92] S. Zhang, Positive line bundles on arithmetic surfaces, Ann. of Math. (2) 136 (1992), 569-587. MR 1189866 (93j:14024)

[Zha95a] S. Zhang, Positive line bundles on arithmetic varieties, J. Amer. Math. Soc. 8 (1995), 187-221. MR 1254133 (95c:14020)

[Zha95b] S. Zhang, Small points and adelic metrics, J. Algebraic Geometry 4 (1995), 281-300. MR.1311351 (96e:14025)

[Zha98] S. Zhang, Equidistribution of small points on abelian varieties, Ann. of Math. (2) 147 (1998), no. 1, 159-165. MR.1609518 (99e:14032)

[Zha06] S. Zhang, Distributions in Algebraic Dynamics, Surveys in Differential Geometry, vol. 10, International Press, Somerville, MA, 2006, pp. 381-430. MR.2408228 (2009k:32016)

Department of Mathematics and Computer Science, University of Lethbridge, LethBRIDGE, AB, T1K 3M4, CANADA

E-mail address: dragos.ghioca@uleth.ca

Department of Mathematics, Hylan Building, University of Rochester, Rochester, NEW YORK 14627

E-mail address: ttucker@math.rochester.edu 